\title{
Potassium Accumulation in Interstitial Space During Epileptiform Seizures
}

\author{
Allen P. Fertziger and James B. Ranck, Jr. ${ }^{1}$ \\ Department of Physiology, The Unizersity of Michigan, Ann Arbor, Michigan 48104 \\ Received November 17, 1969
}

The $\mathrm{K}^{42}$ surface efflux from the cat neocortex and rat hippocampus was studied after preloading with $\mathrm{K}^{42}$ from the surface. This $\mathrm{K}^{42}$ efflux increases from two to nine times during or after all electrically or Metrazol-induced seizures and "spontaneous" seizures. The $\mathrm{K}^{42}$ influx does not change during a seizure, so this increased efflux is not due to change in the diffusion constant or water flow from brain. The time course of the efflux is not compatable with the efflux simply idicating increased turnover of potassium from cells. We conclude there is an increase in the concentration of potassium in the interstitial space during seizures. A model is proposed in which this potassium accumulation is an important step in the regenerative, all-or-none-aspect of the initiation of seizures.

\section{Introduction}

There is a net movement of potassium ions out of neurons and into the interstitial space associated with each action potential. Indeed, the concentration of interstitial potassium, $[\mathrm{K}]_{0}$, increases in the vicinity of active neurons in some circumstances $(3,7,11,19,20,30)$.

In epileptiform seizures there is very rapid firing of many neurons and one might expect the $[\mathrm{K}]_{0}$ to increase. We show that this is, indeed, the case. Spreading depression of Leao is the only case of increased $[\mathrm{K}]_{0}$ with increased neural activity for which there is much evidence that the increased $[\mathrm{K}]_{0}$ is of functional significance (9). We shall suggest that a similar potassium mechanism exists in both phenomena. A preliminary report of this work has alreadly appeared (6).

\section{Methods}

Changes in $[\mathrm{K}]_{0}$ have been measured by the method of Brinley, Kandel and Marshall (2). This method consists of loading a brain with radioactive

1 This work was supported by Grant NB-04352 and GM-00353 from the National Institutes of IIealth. Dr. Fertiziger's present address is Department of Anatomy, Albert Einstein College of Medicine, New York, N.Y. 10461. 
potassium, $\mathrm{K}^{42}$, and using the efflux of this isotope from the brain surface as an index of changes in $[\mathrm{K}]_{0}$. An evaluation of this method will be given later in this paper.

All experiments were performed on cats and rats of both sexes, anesthetized with either sodium pentobarbital or chloralose. After surgical exposure, the pial surface of the cat neocortex or the ventricular surface of the rat hippocampus was incubated for 1-2 hours with radioactive potassium in a prewarmed artificial cerebrospinal fluid. The specific activity of the $\mathrm{K}^{42}$ was $13.9 \mu \mathrm{c} / \mu$ mole $\mathrm{K}^{+}$. In rats, a skin flap was used to retain the column of fluid over the brain, while a Plexiglas well, press fitted into a $12.5-\mathrm{mm}$ diameter trephine hole, was used in the cat. At the end of the incubation period the brain was washed with nonradioactive cerebrospinal fluid to remove any isotope adhering to the pial surface. A chamber, shown in Fig. 1, equipped with stimulating and recording electrodes was then positioned onto $0.25 \mathrm{~cm}^{2}$ of the brain surface. A constant flow of warmed nonradioactive artificial cerebrospinal fluid was perfused through this chamber at a rate of $0.821 \mathrm{ml} / \mathrm{min}$, equal to 21 times the volume of the chamber per minute. The artificial cerebrospinal fluid was $0.5 \mathrm{~mm} \mathrm{Na}_{2}$ $\mathrm{HPO}_{4}, 25 \mathrm{~mm} \mathrm{NaHCO}, 125 \mathrm{~mm} \mathrm{NaCl}, 1.1 \mathrm{~mm} \mathrm{CaCl}, 0.8 \mathrm{~mm} \mathrm{MgCl}_{2}$, and $3.0 \mathrm{~mm} \mathrm{KCl}$. The effluent from the chamber was collected and assayed for radioactivity. In some experiments the perfusate was collected for fixed periods of time (usually $30 \mathrm{sec}$ ), while in other experiments the

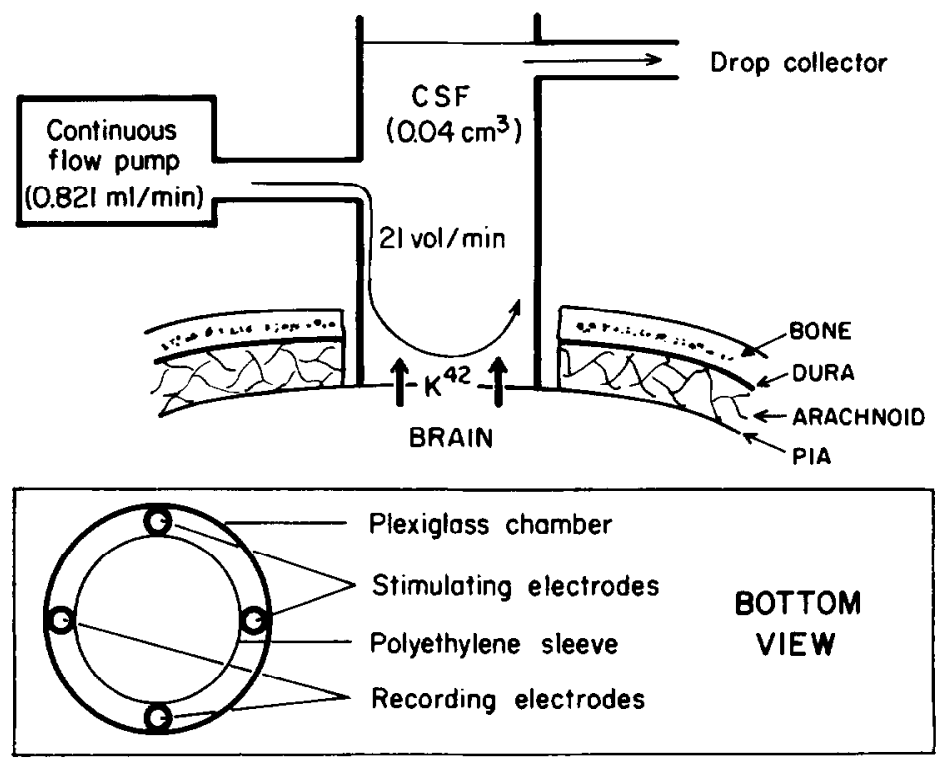

FTG. 1. The perfusion chamber. 
duration of the collection periods was varied. When this was done, it was necessary to determine the volume that was collected, and the radioactivity was expressed as counts per minute per unit volume. The cortical EEG was recorded by a pair of surface electrodes built into the chamber, thus making possible a correlation between perfusate radioactivity and ongoing EEG.

Seizures were elicited either by tetanic stimuli delivered to the brain surface or by the intravenous administration of pentylenetetrazol (Metrazol). When Metrazol was used it was necessary to immobilize animals with gallamine triethiodide (Flaxedil) and give artificial respiration. They, of course, were also under general anesthesia. Figure 2 shows the electrographic patterns of these seizures. We were concerned about the possibility of confusing a seizure with spreading depression; however, in all cases the episode was clearly a seizure. Some seizures had no postictal depression at all.

\section{Results}

The 19 electroshock and Metrazol-induced seizures that have been studied in 13 different animals all resulted in a stereotyped change in the $\mathrm{K}^{42}$ surface efflux. Soon after the start of the seizure, the $\mathrm{K}^{42}$ surface efflux started to rise, reached a maximum, and then returned to the preseizure level. As shown in Table 1, these maxima ranged from 2.0-9.3 times larger than the preseizure efflux (mean $=4.9$ ). No seizure has been studied that has not resulted in this altered $K^{42}$ efflux pattern. In 11 of the 19 seizures that have been studied, the maximum in the $K^{12}$ surface efflux occurred after the seizure was over, and in four of these 11 cases the maximum occurred after the EEG had returned completely to normal (after postictal abnormalities, if any). In the eight remaining seizures

TABLE 1

Maximal $K^{42}$ Surface Effi.ux During Electrically-induced (E) and Metrazol-Induced (M) Sejzures in the Rat Hippocampal Formation (H) and the Cat Suprasyluian Grrus (N)

\begin{tabular}{lcccc}
\hline & \multicolumn{2}{c}{$\begin{array}{l}\text { Peak seizure efflux } \\
\text { Non-seizure efflux }\end{array}$} & \\
Type of seizure & Nean & Range & & No. animals \\
\cline { 2 - 4 } & \multicolumn{2}{c}{ Meares } & \\
\hline H-E & 3.5 & $2.0-6.6$ & 7 & 3 \\
H-M & 8.4 & $7.5-9.2$ & 2 & 2 \\
N-E & 4.8 & $2.3-8.0$ & 4 & 2 \\
N-M & 6.5 & $4.5-9.3$ & 3 & 3 \\
Spont. $(\mathrm{H})$ & 4.3 & $2.9-6.7$ & 3 & 3 \\
& Totals 4.9 & $2.0-9.3$ & 19 & 13 \\
\hline
\end{tabular}



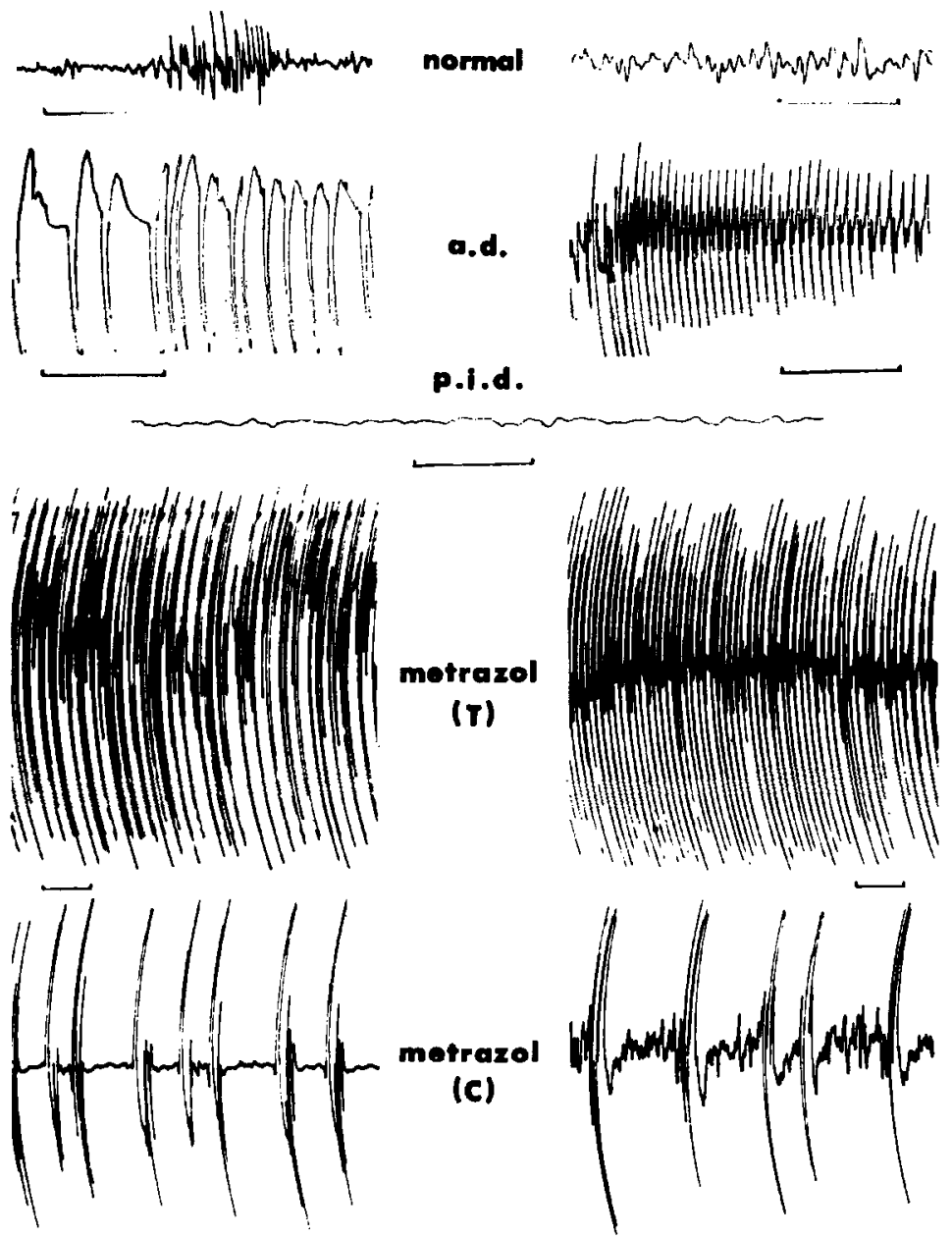

Fig. 2. Typical EEG patterns in neocortex (left) and hippocampus (right).

Normal: a barbiturate spindle is depicted in the neocortical record; a.d.: afterdischarge following electrical stimulation; p.i.d.: postictal depression; $\mathrm{T}$ : tonic phase of a Metrazol seizure; C: clonic phase of a Metrazol seizure. Time calibration 1 sec.

that were studied, the collection periods were too long to determine if the maximum occurred before, during, or after the end of the seizure.

Electroshock Seisures. Figure 3 shows the results of a typical experiment in which the changes in the $\mathrm{K}^{42}$ surface efflux are depicted. In this experiment electroshock was used to elicit an afterdischarge lasting 18 sec in the rat hippocampal formation. (In this figure and in all subsequent figures the circles have been placed at the end of the collection period.) 


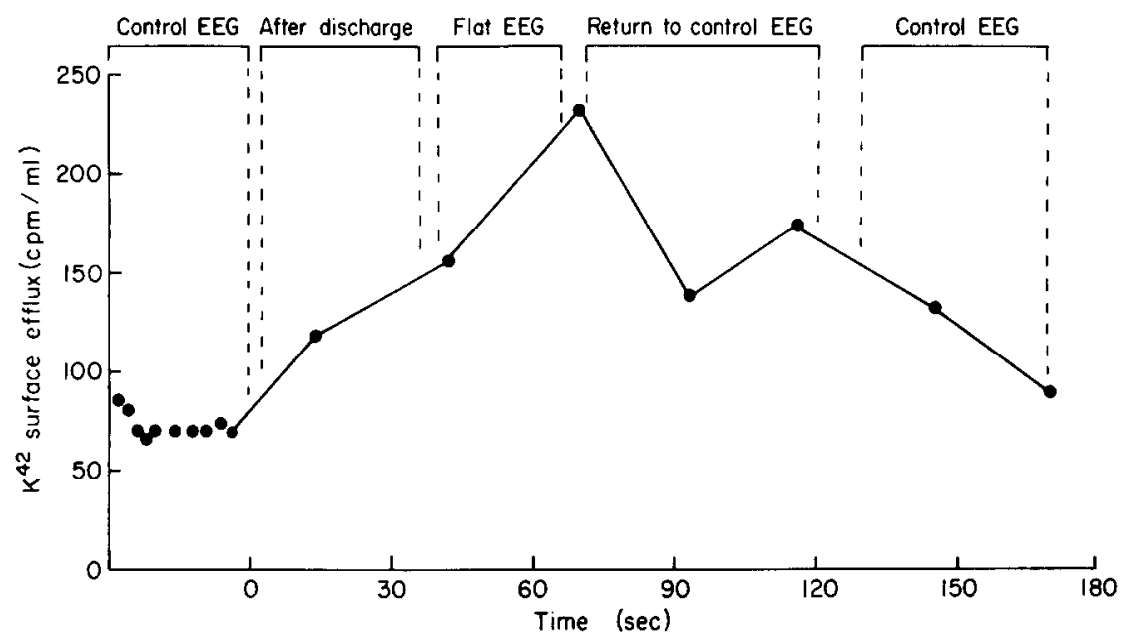

Fig. 3. Effect of an electrically induced seizure on the $\mathrm{K}^{42}$ surface efflux from the cat hippocampal formation. The electrical stimulation was $3 \mathrm{v}, 50 / \mathrm{sec}, 1-\mathrm{msec}$ duration pulses, and occurred at time zero. The time scale for the control efflux periods is compressed. Sample radioactivity is plotted at the end of the collection period.

The $\mathrm{K}^{42}$ surface efflux, which had been quite stable prior to the seizure, started to increase during the seizure, reached a maximum at the end of the postictal depression that followed the afterdischarge, and returned to the preseizure level approximately $3 \mathrm{~min}$ after the onset of the seizure.

When the $K^{42}$ surface efflux was measured during subseizure electrical stimulation in both the rat hippocampal formation and cat neocortex, it consistently remained unchanged from the prestimulus level. In these experiments the only stimulus parameter to be changed was the stimulating voltage. Stimulating frequency and pulse duration remained constant. Voltages used were always more than half the value of the lowest voltage capable of eliciting an afterdischarge. The fact that there does not appear to be a graded relationship between $\mathrm{K}^{+2}$ surface efflux and brain stimulation strongly suggests that the efflux measurements reveal something about the seizure process and not merely that the brain has been stimulated.

Metrazol Seizures. In general, the seizures produced by Metrazol lasted much longer than those produced by electroshock. The tonic phase lasted 19-30 sec and was followed by a clonic phase that continued for as long as 10 min, gradually giving rise to a more normal EEG. Associated with Metrazol seizures in both cat neocortex and rat hippocampal formation is an increase in the $K^{ \pm 2}$ surface efflux (Fig. 4), similar to that seen after electroshock. However, this increase differed in three major ways from that seen after electroshock. First, the $\mathrm{K}^{43}$ surface efflux increased to a 
maximum that was larger than the maximum seen after most electroshock seizures. Secondly, this maximum was always observed to occur much later than in electroshock seizures. In one experiment the maximum occurred 4 min after the seizure had started while in the majority of experiments it occurred between 1.5 and 3 min after the initiation of the seizure. In Fig. 4 the maximum occurs after a 1.5 -min latency. In all cases, the maximum occurred during the clonic phase of the seizure (i.e., never during the tonic phase). Finally, the duration of the elevated $K^{42}$ surface efflux is also much greater than the increase seen after electroshock. The fact that the $\mathrm{K}^{42}$ surface efflux maximum was larger and the duration longer is not surprising inasmuch as the Metrazol seizure was clearly of much longer duration. The longer latency was somewhat surprising, and no clear explanation can be given for it. It may be because the source of the increased $\mathrm{K}^{42}$ surface efflux in electroshock is closer to the brain surface than in a Metrazol seizure. The time course of the $K^{42}$ surface efflux increase shown in Fig. 4 (rat hippocampal formation) is typical for all Metrazol seizures cxcept for the fact that the return of the $\mathrm{K}^{42}$ surface efflux is somewhat longer than is usually seen. This may be related to the fact that the return of the EEG to its preseizure character is also delayed.

Spontaneous Seizures. On three separate occasions, all in rat hippocampal formation, a seizure occurred which had not been directly elicited by brain stimulation. These spontaneously occurring seizures always ap-

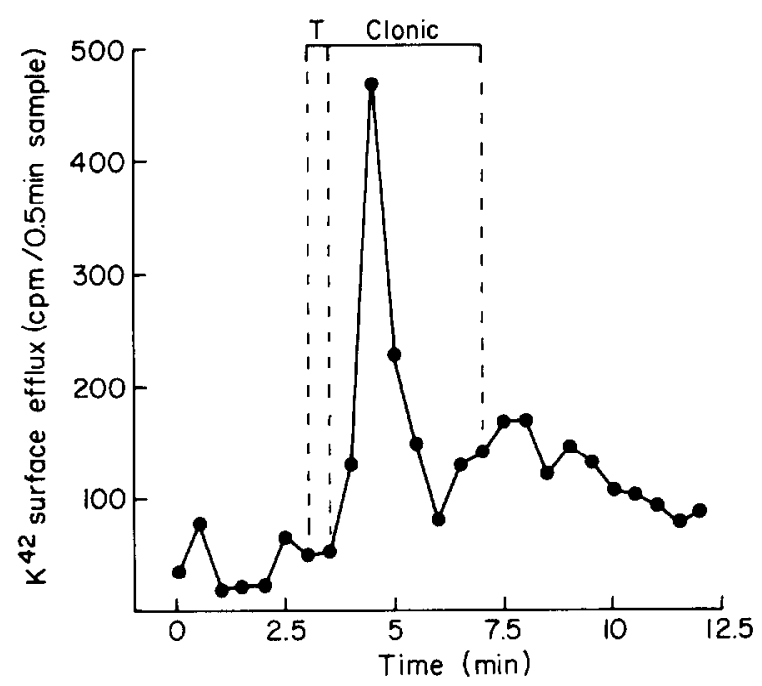

Fig. 4. Effect of a Metrazol-induced seizure on the $\mathrm{K}^{42}$ surface efflux from the cat suprasylvian gyrus. " $T$ " is the tonic phase. Dosage $230 \mathrm{mg} / \mathrm{kg}$. Sample radioactivity is plotted at the end of the collection period. 
peared within 5 min after an electrically induced seizure and for this reason the word "spontaneous" should be used with caution. However, these seizures always occurred well after the effects of the previous afterdischarge appeared to be over and a normal EEG resumed. In fact, in two of the three cases, the $\mathrm{K}^{* 2}$ surface efflux had completely returned to preseizure leveis, while in the third case the return was almost conplete. All three of these so-called spontaneous seizures were associated with an increase in $K^{42}$ surface efflux that was indistiguishable from electroshock in terms of magnitude and time course. Spontaneous seizures were never observed in neocortex. Finding them in the hippocampus is not wholly unexpected in light of the low seizure threshold demonstrated by this structure.

\section{Evaluation of the $K^{\ddagger 2}$ Surface Efflux Method}

The method of Brinley, Kandel and Marshall (2) that has been described represents an indirect approach to studying the dynamics of the interstitial potassium environment of the brain. We cannot draw any conclusions from it without the following considerations and experiments.

A negligible amount of $\mathrm{K}^{42}$ that reaches the surface of the brain will enter the surface perfusate directly by moving across cell membranes at the brain surface. Virtually all will diffuse into the perfusate via the interstitial space. By Fick's first law of diffusion, flux is equal to the concentration gradient in the interstitial space times the effective diffusion constant of the brain. ${ }^{2}$ An increased surface efflux can therefore be due either to an increased concentration gradient at the surface, an increase in the effective diffusion constant at the surface, or to a bulk flow of fluid from the surface. We have eliminated the possibility of an increased diffusion constant or bulk flow of fluid from the surface by demonstrating that the rate of $i n$ $f u x$ or $\mathrm{K}^{42}$ from the ventricular surface into the hippocampal formation during a seizure is the same as the nonseizure influx. The results of this experiment are summarized in Table 2 . Influx was determined by measuring the specific activity of excised pieces of hippocampus after a 2-min exposure to $\mathrm{K}^{42}$ on the surface, with and without seizures produced by surface stimulation. An increased effective diffusion constant should increase the influx (and increase efflux). A bulk flow of fluid out should decrease the influx (and increase efflux). We consider the possibility of both factors having an equal and opposite effect on influx sufficiently unlikely so as to be ignored. Therefore, this increased $\mathrm{K}^{42}$ surface efflux must be a result of an increased interstitial $\mathrm{K}^{42}$ concentration.

2 Effective diffusion constant includes factors expressing the diffusion constant of the interstitial space, size, and geometry of the space, plus any diffusional barriers such as pia mater. 
TABLE 2

A Comparison of the $K^{42}$ Influx into Brain During Seizures and Non-SEIzURE ACtivity

Specific activity of the incubation medium was the same in all cases

\begin{tabular}{ccc} 
Expt. & $\begin{array}{c}\text { Duration } \\
\text { Incubation } \\
(\text { min })\end{array}$ & $\begin{array}{c}\text { Spec. Activity } \\
\text { Brain } \\
\left(\mathrm{cpm} / \mathrm{meg} \mathrm{K}^{+}\right)\end{array}$ \\
\hline Control & 2 & $1.52 \times 10^{-6}$ \\
Control & 2 & $1.42 \times 10^{-6}$ \\
Seizure & 2 & $0.49 \times 10^{-6}$ \\
Seizure & 2 & $2.05 \times 10^{-6}$ \\
Seizure & 2 & $1.47 \times 10^{-6}$ \\
\hline
\end{tabular}

However, does an increase in interstitial $\mathrm{K}^{-42}$ indicate an increase in interstitial, nonradioactive potassium? If the specific activity of interstitial potassium (S. A.o) is significantly smaller than the intracellular specific activity $\left(\mathrm{S} . \mathrm{A}_{. \mathrm{i}}\right.$ ) (ignoring any bound potassium), then a one-way efflux of potassium from cells into a $\mathrm{K}^{42}$-depleted interstitium could produce an increased surface efflux without much elevation of the interstitial potassium concentration. Perfusing the brain surface with non-radioactive cerebrospinal fluid will cause a reduction of the S.A.o in superficial layers, but how deep will this be? S.A.o will not fall below $S_{.} A_{{ }_{i}}$ in regions where the time constant for the rate of $\mathrm{K}^{42}$ turnover by transmembrane fluxes is less than the time needed to reach the brain surface by diffusion. The rate of $\mathrm{K}^{42}$ turnover in neocortex can be approximated by assuming a resting transmembrane potassium flux of $14 \times 10^{-12} \mathrm{moles} / \mathrm{cm}^{2} \mathrm{sec}$, an interstitial space due to cell separation of $700 \AA$, an interstitial potassium concentration of $4 \mathrm{~mm}$, and that two-thirds of the membranes are glial with no transmembrane flux. ${ }^{3}$ With these assumptions, interstitial $K^{42}$ will be expected to turn over with a time constant of $3 \mathrm{sec}$. Consequently, S.A.o will fall below S.A.i only in those regions where the diffusion time for potassium to reach the surface is less than $3 \mathrm{sec}$. Obviously this will comprise only the most superficial elements of the cortical mantle. If this increased surface efflux is coming only from superficial elements, there will

\footnotetext{
3 These values were selected so as to bias this argument against a rapid turnover time constant and not to indicate our personal preference for these values. The value for resting transmembrane potassium flux was obtained from flux measurements on sepia axons (13). This value seems reasonable because the specific membrane resistance of sepia axons is comparable to the resistance of mammalian neurons. In fact, the increased neuronal activity that takes place during seizures suggests a larger transmembrane potassium flux than the value we have chosen. The quantitative contribution of neuroglia cells to the potassium turnover is less clear and therefore has been completely excluded from this argument, although it is surely important.
} 
be a delay of less than $3 \mathrm{sec}$ between $K^{42}$ efflux from cells and its collection on the surface. On the other hand, if the increased $\mathrm{K}^{42}$ efflux at the surface is associated with increased potassium in depth, then the maximal surface efflux would be expected to occur after a considerably longer delay. Since the surface efflux maximum occurs after the end of the seizure and in several cases after the return of a normal EEG, the hypothesis that superficial elements are responsible for the $\mathrm{K}^{42}$ surface efflux maximum scems untenable and can be rejecter. (The case is especially strong in the hippocampus, because of the alveus, a predominantly myelinated fiber tract that runs along the ventricular surface of the hippocampus. In this myelinated fiber tract ionic fluxcs occur only across a small fraction of the total membrane surface, the nodes of Ranvier. Therefore, in the alveus both the surfaces contributing to the increased $\mathrm{K}^{ \pm 2}$ efflux from cells and those contributing to the equilibration of $S . A_{i}$ and $S . A_{. o}$ are decreased.) The large fluxes of both $\mathrm{K}$ and $\mathrm{K}^{42}$ occur in gray matter in depth. We, therefore, conclude that the increased surface efflux is an indication of an increased potassium concentration in the interstitial space of the brain. While the magnitude of this accumulation cannot be determined from these data, it seems likely that it is at least as large as the increase in the $\mathrm{K}^{42}$ surface efflux.

\section{Discussion}

We have shown an increased $\mathrm{K}^{42}$ surface efflux to be associated with three types of seizures in two places without any exceptions. There seems to be little question that this phenomenon is a characteristic of all epileptiform seizures. The observation that there is an increase in the $[\mathrm{K}]_{0}$ of mammalian brain during epileptiform activity is not totally unexpected. What is surprising is that this change is not a trivial one in amount or duration but is comparable to the increased efflux of three to eight times in $[\mathrm{K}]_{0}$ of several minutes duration reported by Brinley, Kandel and Marshall (3), during spreading depression of Leao.

Because of the magnitude of the increase in $[\mathrm{K}]_{0}$, it might be argued that a special mechanism for elevating $[\mathrm{K}]_{\circ}$ is necessary. However, a simple calculation relating the magnitude of the potassium efflux associated with the size of the brain's interstitial space reveals that this is not necessary. Arbitrarily assuming an interstitial space of $300 \AA$ surrounding a neuron, the potassium flux that is associated with each action potential ( $5 \mathrm{pmole} / \mathrm{cm}^{2} /$ action potential) elevates the $[\mathrm{K}]_{0}$ by $2 \mathrm{~mm} / 1$, provided that the released ions do not diffuse away, which normally occurs. If, however, all the neurons in an area fire simultaneously, the places potassium can diffuse to are markedly reduced.

One cannot decide whether the accumulation of potassium ions in brain 
interstitium is causally related to the seizure process, as has been suggested previously by Green (10), or if it merely is a "by-product" of the seizure. The fact that the maxinum $\mathrm{K}^{42}$ efflux can occur after the seizure does not prove the $[\mathrm{K}]_{0}$ accumulation is only a by-product of the seizure, because, as we have shown above, there is a substantial and unknown delay between $[\mathrm{K}]_{0}$ increase and $\mathrm{K}^{42}$ efflux. However, we shall develop here the hypothesis that an increase in $[\mathrm{K}]_{.}$is of causal significance in the seizure and show that this fits fairly well with many electrophysiological facts about seizures.

An increase in the $[\mathrm{K}]_{\mathrm{o}}$ of brain will depolarize neurons, which will make them more excitable. The increase in $[\mathrm{K}]_{\text {o }}$ will also shift the equilibrium potential of potassium in a depolarizing direction. Since the neuron is partially depolarized, chloride, if passively distributed across the cell membrane, will enter the neuron, and the chloride equilibrium potential will also be shifted in a depolarizing direction. The shift of the potassium and chloride equilibrium potentials in a depolariziing direction will cause the reversal potential of the IPSP to move in a depolarizing direction from what it was before (not necessarily to cause a depolarizing IPSP), and the efficacy of post-synaptic inhibition will be reduced. This is a second factor increasing the excitability of the postsynaptic neuron. Because of increased excitability, the neuron will increase its rate of firing, causing a further increase in $[\mathrm{K}]_{\mathrm{o}}$, so the process is regenerative. Eventually the depolarization due to increased $[\mathrm{K}]_{0}$ may reach threshold, directly causing and maintaining firing beyond any effect from excitatory synaptic input, although spike activity need not always occur due to accommodation. If this depolarization is sustained and large enough the neuron will be silenced due to cathodal block or to changes in the spike mechanism. Sodium is entering the neuron with action potentials and chloride is entering passively due to a sustained depolarization so water can be expected to follow, causing the neuron to enlarge. Presumably this will decrease the size of the interstitial space, and therefore further increase $[\mathrm{K}]_{0}$ and also increase the electrical impedance of brain. Surely, increased $[\mathrm{K}]_{0}$ has other effects also, but those just described seem most likely and, as will be shown below, can be compared with published data.

Note that this hypothesis says nothing about the initial increase in excitability or how the $[\mathrm{K}]_{0}$ became elevated initially, the underlying pathology, the interictal episodes, or the spread of a seizure, and only weakly relates to its termination. The major thrust of this proposed potassium mechanism is in the regenerative all-or-none aspect of the initiation of seizures--when does an interictal episode become ictal? This hypothesis is summarized in Fig. 5. Up to some value of $[\mathrm{K}]_{0}$ the process does not become regenerative, and is only a localized or subthresholit effect-the fac- 
tors decreasing $[\mathrm{K}]_{\circ}$ (diffusion in interstitial space and movement into cells) being more effective than those increasing it. At some threshold $[\mathrm{K}]_{0}$ value, however, the mechanism becomes regenerative and a seizure is initiated.

To our knowledge there are eight stndies (from six laboratories) of intracellular electrical events during ictal phenomena, using Metrazol- and penicillin-induced seizures, and afterdischarges $(4,12,18,26-29,31)$. In seven of these studies most neurons had a sustained depolarization of many seconds and many millivolts during the seizure $(4,12,18,27-29$, 31). (Let us call this "sustained profund depolarization.") Rapid firing of ncurons directly from the sustained profound depolarization occurred until the depolarization became so great that the shape of action potentials became abnormal and then were no longer initiated. However, in any one study not all neurons showed these sustained profound depolarizations. Furthermore, in these reports there are two situations in which sustained profound depolarizations were not common. In one of these exceptions (12) there was sustained profound depolarization of most hippocampal pyramidal neurons during a seizure if the fornix was intact. but if the fornix had been previously cut and allowed to degenerate, most of the hippocampal pyramidal neurons showed hyperpolarization during a seizure and none depolarized. The other exception (26) is in hippocampal seizure initiated by subiculum stimulation in which almost no cells with sustained profound depolarization were seen in hippocampus.

During sustained profound depolarizations seen during seizures all transient hyperpolarizations were reported to decrease or disappear in some studies $(4,18,27-29,31)$.

Profound depolarizations of greater than a second have not been seen in any of the manty intracellular studies of interictal discharge $(1,4,8$,

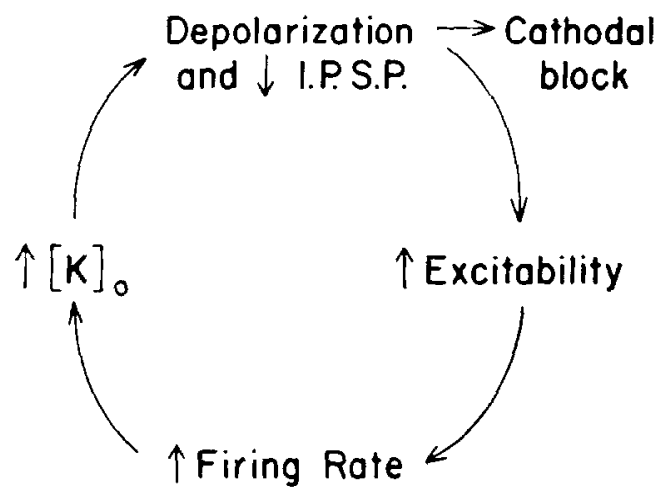

FIG. 5. Regenerative consequences of accumlation of interstitial $\mathrm{K}+$. 
$14-17,21-25,27-29,31,33)$. Most transient hyperpolarizations did not decrease or disappear in any of these same studies of interictal discharge.

The electrical impedance of brain increases during a seizure $(5,32)$. Neither of these reports on impedance describes the relation to interictal abnormalities, from which we assume there was little change.

Thus three kinds of electrophysiological changes which the potassium accumulation hypothesis predicts would occur during seizures but not during interictal abnormalities have indeed been seen during seizures and not during interictal abnormalities. Importantly though, some neurons in a seizure do not show the kinds of electrophysiological changes simply predicted by the potassium accumulation hypothesis. Sawa, Nakamura and Naito (29) and Matsumato and Ajmone-Marsan (18) attempted to explain these "exceptions" in their own data and in others in terms of synaptic inhibition by rapidly firing cells elsewhere or elimination of synaptic input. The argreement of the predictions of the hypothesis and published results seems good enough so that the hypothesis must be seriously considered. Since there are many other possible explanations for these electrophysiological data, they are only consistent with the hypothesis and the hypothesis is not proved.

The hypothesis is similar to the commonly held hypothesis of spreading depression. Since the same structure can have both seizures and spreading depression, which are grossly different phenomena, there are clearly other major differences in their mechanisms.

An essential part of this regenerative potassium accumulation hypothesis is the idea that there exists an interstitial potassium concentration which, when exceeded, triggers the regenerative all-or-none process depicted in Fig. 5. As a test of this aspect of the hypothesis and to gain a better understanding of how the brain handles high interstitial potassium concentrations, we have attempted to vary $[\mathrm{K}]_{\text {o }}$ by varying the surface $[\mathrm{K}]$ and examine the effects of this on the $\mathrm{K}^{42}$ surface efflux and EEG.

We have measured the $\mathrm{K}^{12}$ surface efflux from the surface of the cat suprasylvian gyrus while perfusing the surface of the brain within and surrounding the chamber described earlier with different concentrations of potassium. Figure 6 shows the results of a typical experiment. The surface $[\mathrm{K}]$ was varied between the normal cerebrospinal fluid $[\mathrm{K}], 3 \mathrm{~mm}$ and $120 \mathrm{~mm}$, while the perfusate was collected for 1 -min periods. Isotonicity was maintained by replacing sodium with equimolar amounts of potassium. The $[\mathrm{K}]$ in the perfusate was varied in a stepwise fashion consisting of 10-15 min exposures to each different solution. A 10-15 min exposure to normal cerebrospinal fluid was spaced between each high potassium exposure. The same experiment was repeated in three different cats, all with the same results. In all experiments, the $\mathrm{K}^{42}$ surface efflux behaved in an 

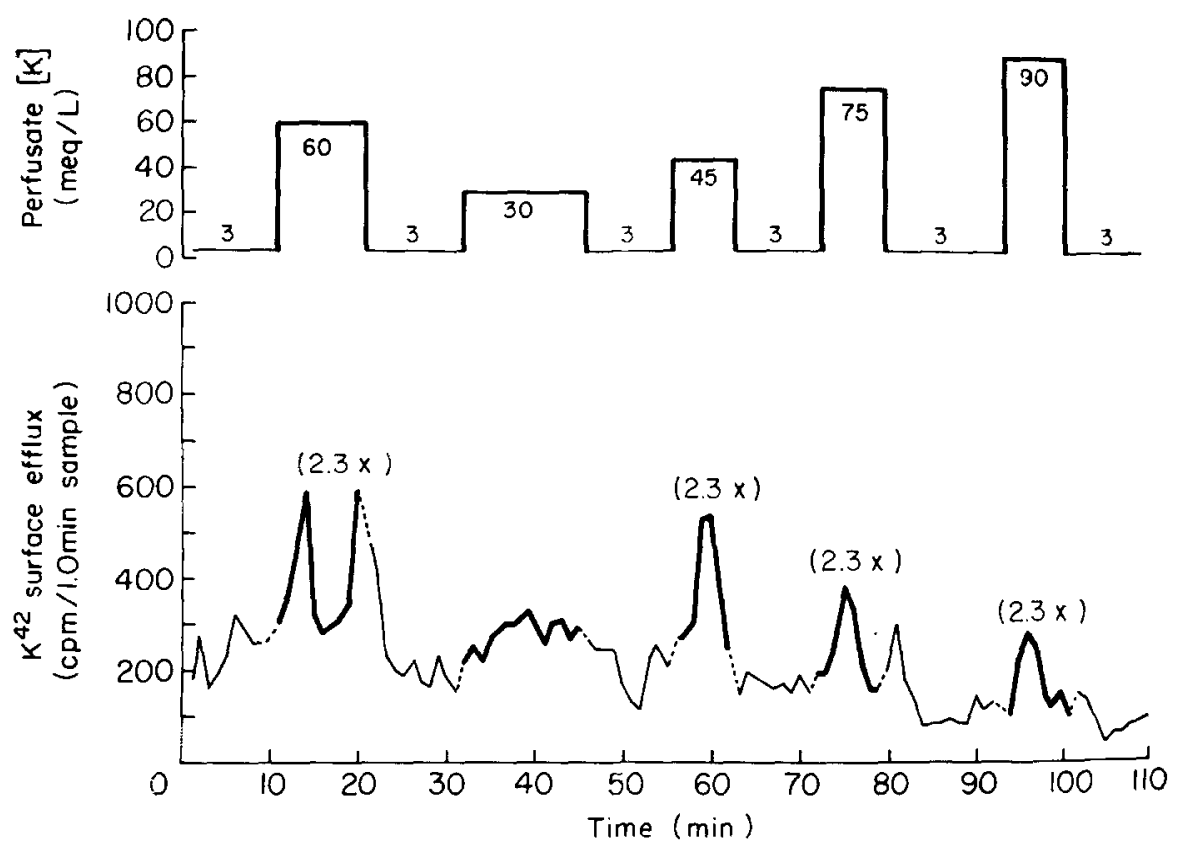

Fig. 6. Effects of perfusing the surface of the cat suprasylvian gyrus with varying concentrations of potassium, on the $K^{42}$ surface efflux from that structure. The perfusate $[\mathrm{K}$ ] was increased by replacing $\mathrm{Na}$ with $\mathrm{K}$. The heavy line of the effux record corresponds to periods of abnormal perfusate with the ratio of the maximal efflux to the preseizure efflux shown. (Note the declining baseline which is probably a manifestation of depletion of brain radioactivity during a perfusion period lasting 110 min.).

all-or-none fashion, regardless of the $[\mathrm{K}]$ of the perfusatc. $\Lambda \mathrm{t}$ concentrations up to $45 \mathrm{~mm}$ (and in some cases $60 \mathrm{~mm}$ ) neither the $\mathrm{K}^{42}$ surface efflux nor the EEG changed. However, beyond these concentrations, which are 15-20 times greater than normal cerebrospinal fluid $[\mathrm{K}]$, the $\mathrm{K}^{12}$ surface efflux underwent the characteristic change. This is shown in Fig. 6.

The effux changes were always paralleled by a marked change in the EEG, either flattening or spiking. The changes were not epileptiform seizures. It is likely that this was spreading depression, but $\mathrm{d}-\mathrm{c}$ measurements were not made, so we do not have a definite identification. The changes in $K^{42}$ surface efflux and EEG evoked by high potassium on the brain surface were of limited duration, even when the abnormal perfusate continued. Sometimes, as in the first and fourth $(60$ and $75 \mathrm{~mm})$ test in Fig. 5, more than one episode was triggered. The latency from onset of high potassium to the beginning of an abnormal episode was up to 2.7 min and variable. 
The fact that there is no change in either the $\mathrm{K}^{42}$ surface efflux or the EEG at surface concentrations 15-20 times greater than normal suggests that the brain is able to handle these potassium loads without a change in the $[\mathrm{K}]_{0}$. However, at higher surface potassium concentrations, this control appears to break down and interstitial potassium undergoes a large change as indicated by the stereotyped increase in $\mathrm{K}^{42}$ surface efflux and the change in EEG. A high CFS $[\mathrm{K}]$ has been shown to result in seizures in hippocampus (34).

\section{References}

1. Atkinson, J. R., and A. A. WARD, JR. 1964. Intracellular studies of cortical neurons in chronic epileptogenic foci in the monkey. Exptl. Neurol. 10: 285295.

2. Brinley, F. J., E. R. Kandei, and W. H. Marshall. 1960a. Effect of gamma amino butyric acid (GABA) on $\mathrm{K}^{42}$ outflux from rabbit cortex. J. Neurophysiol. 23 : 237-245.

3. Brinley, F. J., E. R. Kandel, and W. H. Marshal.l. 1960b. Potassium outflux from rabbit cortex during spreading depression. J. Neurophysiol. 23 : 246-256.

4. Creutzfeldt, O. D., S. Watanabe, and H. D. Lux. 1966. Relations between EEG phenomena and potentials of single cortical cells. II. Spontaneous and convulsoid activity. Electroencephalogr. Clin. Neurophysiol. 20: 19-37.

5. Elazar, Z., R. T. Kado, and W. R. AdEY. 1966. Impedance changes during epileptic seizures. Epilepsia $7: 291-307$.

6. FertZiger, A. P., and J. B. RANCK, JR. 1968. An increase in brain interstitial potassium during three types of epileptiform activity as measured by $\mathrm{K}^{42}$ efflux. Fed. Proc. $27: 976$.

7. Frankenhaueser, B., and A. L. Hodgrin. 1956. The after-effects of impulses in the giant nerve fibers of Loligo. $J$. Physinl. London 131: 341-376.

8. Goldensohn, E. S., and D. P. Purpura. 1963. Intracellular potentials of cortical neurons during focal epileptogenic discharges. Science 139: 840-842.

9. Grafstein, B. 1963. Neural release of potassium during spreading depression, pp. 87-124. In "Brain Function, Cortical Excitability and Steady Potentials, Relations of Basic Research to Space Biology." (UCLA Forum in Medical Sciences, Number 1.) M. A. B. Brazier [ed.]. University of California Press, Berkeley and Los Angeles.

10. Green, J. D. 1964. The hippocampus. Physiol. Rea. $44: 561-608$.

11. Greengard, P., and R. W. Straub. 1958. After-potentials in mammalian nonmyelinated nerve fibers. J. Physiol. London $144: 442-462$.

12. Kandel, E. R., and W. A. Spencerr. 1961. Excitation and inhibition on pyramidal cells during hippocampal seizure. Exp. Neurol. 4: 162-179.

13. Keynes, R. D. 1951. The ionic movements during nervous activity. J. Physiol. London 114: 119-150.

14. Lr, C. L. 1959. Cortical intracellular potentials and their responses to strychnine. J. Neuraphysiol. 22 : 436-450.

15. Lr, C. L., S. N. Chou, and S. Y. How ARd. 1961. Basic mechanisms of single cell discharge in the cerebral cortex. Epilepsia 2 : 13-21.

16. Matsumoto, H. 1964. Intracellular events during the activation of cortical epileptiform discharges. Electroencephalogr. Clin. Neurophysiol. 17: 294-307. 
17. Matsumoto, H., and C. Ajmone-Marsax. 1964a. Cortical cellular phenomena in experimental epilepsy: interictal manifestations. F.1 7 . Notrol. 9: 286-304.

18. Matsumoto, H., and C. Ajmone-Marsan. 1964b. Cortical cellular phenomena in experimental epilepsy: ictal manifestations. F.rp. Nourol. 9: 305-326.

19. Naramashi, T., and T. Yamasaki. 1960. Mechanisn of the after-potential production in the giant axons of the cockroach. J. Physiol. London 151: 7588.

20. Orkann, R. K., J. G. Nicholls, and S. W. Kllflr. 1966. The effect of nerve impulses on the membrane potential of glial cells in the central nervous system of amphibia. J. Neturophysiol. 29 : 788-806.

21. Pollen, D. A., and C. Ajome-Marsan. 1965.Cortical inhibitory postsynaptic potentials and strychninization. J. Neurophysiol. 28 : 342-358.

22. Prince, D. A. 1968a. Inhibition in "epileptic" neurons, E.xp. Neurol. $21: 307-321$.

23. Prince, D. A. 1968b. The depolarization shift in "epileptic" neurons. Exp. Neurol. 21 : 467-485.

24. Prince, D. A. 1969. Electrophysiology of "epileptic" neurons: spike generation. Electroencephalogr. Clin. Neurophysiol. $26: 476-487$.

25. Prince, D. A., and K. J. Futamachi. 1968. Intracellular recordings in chronic focal epilepsy. Brain Res. $11: 681-684$.

26. Purpura, D. P., J. G. McMurtry, C. F. Leonard, and A. Malliani. 1966. Evidence for dendritic origin of spikes without depolarizing prepotentials in hippocampal neurons during and after seizure. J. Newrophysiol. 29 : 954-979.

27. Sawa, M., N. Maruyama, and S. Kajr. 1963. Intracellular potential during electrically induced seizures. I:lectrochcophaloyr. Clin. Neurophysiol. 15: 209220.

28. SAWA, M., S. KAJI, and K. Usuki. 1965. Intracellular phenomena in electrically induced seizures. Electrocncephalogr. Clin. Nourophysiol. 19: 248-255.

29. Sawa, M., K. Nakamura, and H. Naito. 1968. Intracellular phenomena and spread of epileptic seizure discharges. Electrocncephaloyr. Clin. Nourophysiol. 24: $146-154$.

30. Shanes, A. M. 1951. Potassium movement in relation to nerve activity. J. Gen. Physiol. 34 : 795-807.

31. Suguya, E., S. Goloring, and J. L. O'Lenry. 1964. Intracellular potentials associated with DCR and seizure discharge in cat. Eloctrocnicphalogr. Clin. Neurophysiol. 17 : 661-669.

32. Van Harreveld, A., and J. P. Schade. 1962. Changes in electrical conductivity of cerebral cortex during seizure activity. E.r. Ncurol. $5: 383-400$.

33. Wildfr, B. J., and F. Morrell. 1967. Cellular behavior in secondary epileptic lesions. Neurology 17 : 1193-1204.

34. Zuckerman, E. C., and G. H. Glaser. 1968. Hippocampal epileptic activity induced by localized ventricular perfusion with high potassium cerebrospinal fluid. Exp. Neurol. $20: 87-110$. 